\title{
Optical Variability of the $\lambda$ Eri Star HD 105382: Pulsation or Rotation? ${ }^{1}$
}

\author{
M. Briquet \\ Institut d'Astrophysique et Géophysique, Université de Liège, Belgium \\ C. Aerts, P. De Cat \\ Instituut voor Sterrenkunde, Katholieke Universiteit Leuven, Belgium
}

\begin{abstract}
HD 105382 is clearly a monoperiodic, very active $\lambda$ Eri star with the frequency $0.7721 \mathrm{~d}^{-1}$. Its photometric behaviour and the moment variations are not typical for a standard pulsational model.
\end{abstract}

We have taken a high-resolution $\mathrm{H} \alpha$ spectrum in May 1996, which shows double-peaked emission with a maximum of 7.5 continuum units. We conclude that HD 105382 is a very active $\lambda$ Eri star, despite previous suggestions of its non-Be nature.

Measurements of HD 105382 in the Geneva seven-colour photometric system were obtained in 1997. Besides these, we have at our disposal 105 high-resolution spectra obtained with the CAT/CES during 10 separate weeks of monitoring spread over 1996-1998. Both sets of data reveal a monoperiodic star with the frequency $0.7721 \mathrm{~d}^{-1}$.

We confronted the observations with a nonradial pulsation model by comparing the observed amplitudes for the seven Geneva filters with the theoretically predicted amplitudes (Heynderickx, Waelkens, \& Smeyers 1994). We also compared the first three velocity moments with theoretical predictions (Aerts, De Pauw, \& Waelkens 1992). From these two analyses we make the preliminary conclusion that HD 105382 is not pulsating according to the standard theory.

In the near future we plan to make a mode identification from the spectra. At the same time we will consider a rotational modulation model. This will enable us to determine what kind of model best corresponds to the observations.

\section{References}

Aerts, C., De Pauw, M., \& Waelkens, C. 1992, A\&A, 266, 294

Heynderickx, D., Waelkens, C., \& Smeyers, P. 1994, A\&AS, 105, 447

\footnotetext{
${ }^{1}$ Based on observations obtained with the Swiss photometric telescope and ESO's CAT/CES telescope, both situated at La Silla, Chile.
} 